PROCEEDINGS OF THE

AMERICAN MATHEMATICAL SOCIETY

Volume 125, Number 7, July 1997, Pages 2171-2174

S 0002-9939(97)03843-4

\title{
THE UNIVERSAL NILPOTENT GROUP COMPACTIFICATION OF A SEMIGROUP
}

\author{
H. R. EBRAHIMI-VISHKI AND M. A. POURABDOLLAH
}

(Communicated by Palle E. T. Jorgensen)

\begin{abstract}
The purpose of this paper is to introduce an algebra of functions on a semitopological semigroup and to study these functions from the point of view of universal semigroup compactification. We show that the corresponding semigroup compactification of this algebra is universal with respect to the property of being a nilpotent group.
\end{abstract}

The general approach to the theory of semigroup compactification is based on the Gelfand-Naimark theory of $C^{*}$-algebras of functions. There are many papers which deal with the characterization of certain universal semigroup compactifications in terms of function algebras. Seminal work in this context was done by K. de Leeuw and I. Glicksberg [3]. The universal group compactification is given by Junghenn [6], in terms of some types of distal functions. This paper deals with the construction of a function algebra on a semitopological semigroup, used to characterize the universal nilpotent group compactification of it, and investigating some of its properties.

First we recall some preliminaries. Throughout this paper, $S$ shall be a semitopological semigroup, unless otherwise mentioned. For notation and terminology we shall follow Berglund et al. [2] as far as possible. Thus a semigroup compactification of $S$ is a pair $(\psi, X)$, where $X$ is a compact, Hausdorff, right topological semigroup and $\psi: S \rightarrow X$ is a continuous homomorphism with dense image such that for each $s \in S$, the mapping $x \mapsto \psi(s) x: X \rightarrow X$ is continuous.

The $C^{*}$-algebra of all continuous bounded complex-valued functions on a topological space $Y$ is denoted by $C(Y)$. For $C(S)$ left and right translations $L_{s}$ and $R_{t}$ are defined for all $s, t \in S$ and $f \in C(S)$ by $\left(L_{s} f\right)(t)=f(s t)=\left(R_{t} f\right)(s)$. A left translation invariant $C^{*}$-subalgebra $F$ of $C(S)$ (i.e. $L_{s} f \in F$ for all $s \in S$ and $f \in F$ ) containing the constant functions is called $m$-admissible if the function $s \mapsto\left(T_{\mu} f\right)(s)=\mu\left(L_{s} f\right)$ is in $F$ for all $f \in F$ and $\mu \in S^{F}$ (=the spectrum of $F$ ); then the product of $\mu, \nu \in S^{F}$ can be defined by $\mu \nu=\mu \circ T_{\nu}$ and the Gelfand topology on $S^{F}$ makes $\left(\epsilon, S^{F}\right)$ a semigroup compactification (called the $F$-compactification) of $S$, where $\epsilon: S \rightarrow S^{F}$ is the evaluation mapping. The reader is referred to sections 3.1 and 3.3 of [2] for the one-to-one correspondence between compactifications of $S$ and $m$-admissible subalgebras of $C(S)$, and also for a discussion of universal $P$-compactifications, whose existence for a wide variety of properties $P$ is given in terms of subdirect products.

Received by the editors January 16, 1996.

1991 Mathematics Subject Classification. Primary 22A20, 43A60.

Key words and phrases. Semitopological semigroup, (universal) semigroup compactification, weakly (strongly) almost periodic function, distal function, nilpotent group. 
Still following [2] and also [6], the $m$-admissible algebras of weakly almost periodic, strongly almost periodic, minimal distal and strongly distal functions on $S$ are denoted by $W A P, S A P, M D$ and $S D$, respectively. We write $G P$ for $M D \cap S D$; then the $G P$-compactification of $S$ is universal with respect to the property of being a group, [6, theorem 3.4]. Using Ellis's ( joint continuity ) theorem [4], one can show that $G P \cap W A P=S A P$. The left invariant probability measure on the right topological group $S^{G P}$ (see [7], or [2, Appendix $\left.C, C_{3}\right]$ ) gives a left invariant mean on $G P$. In contrast to the situation for $S A P$, it follows from a remark at the end of [1] that, in general, the left invariant mean on $G P$ is not necessarily unique. The existing examples support the conjecture that the left invariant mean on $G P$ is unique if and only if $G P$ is left introverted (i.e. $T_{\mu} f \in G P$ for all $f \in G P$ and all $\mu$ in the dual of $G P)$. Notice that the function $f(n)=e^{i n^{2}}$ on $(\mathbf{Z},+)$, which is in $G P$, has the indicator function of $\{0\}$ in the pointwise closed convex hull of its set of translates; hence $\operatorname{GP}(\mathbf{Z},+)$ is not left introverted (see also the example at the end of $[7])$.

In what follows, $n$ will denote a fixed arbitrary positive integer and $[., ., \ldots,$.$] is$ the commutator of weight $n+1$, as defined in [8], in the group $\left\{T_{\mu}: \mu \in S^{G P}\right\}$; however in the proof of the next theorem it will be in the group $S^{G P}$. Now, we examine the properties of those functions $f \in G P$ for which

$$
\left[\lim _{\alpha_{1}} R_{s_{\alpha_{1}}}, \lim _{\alpha_{2}} R_{s_{\alpha_{2}}}, \cdots, \lim _{\alpha_{n+1}} R_{s_{\alpha_{n+1}}}\right](f)=f
$$

for arbitrary nets $\left\{s_{\alpha_{k}}\right\}, 1 \leq k \leq n+1$, in $S$, where the involved limits are pointwise. We write $N_{n} G(S)$ for the set of all such functions (but we usually suppress the letter $S$ ). Trivially a function $f \in G P$ is in $N_{n} G$ iff $\left[T_{\mu_{1}}, T_{\mu_{2}}, \cdots, T_{\mu_{n+1}}\right](f)=f$ (equivalently, $\left[T_{\mu_{1}}, T_{\mu_{2}}, \cdots, T_{\mu_{n+1}}\right]\left(T_{\mu} f\right)=T_{\mu} f$ ), for arbitrary elements $\mu_{1}, \mu_{2}, \cdots$, $\mu_{n+1}, \mu$ in $S^{G P}$; the latter is equivalent to the fact that the enveloping semigroup of the flow $\left(S, X_{f}\right)$ (which is obtained from the natural action $(s, g) \mapsto R_{s} g$, where $X_{f}$ is the pointwise closure of $\left\{R_{s} f: s \in S\right\}$ ) is a nilpotent group of class $n$.

The following theorem states the main property of $N_{n} G$.

Theorem. Let $S$ be a semitopological semigroup. Then $N_{n} G(S)$ is an m-admissible subalgebra of $C(S)$, and $N_{n} G$-compactification of $S$ is universal with respect to the property of being a nilpotent group of class $n$.

Proof. The $m$-admissibility of $N_{n} G$ is an immediate consequence of the definition and the discussions preceding the theorem. Let $\mu_{1}, \mu_{2}, \cdots, \mu_{n+1}$ and $\mu$ be arbitrary elements of $S^{G P}$. For each $f \in N_{n} G,\left[\mu_{1}, \mu_{2}, \cdots, \mu_{n+1}\right](f)=e(f)$, in which $e$ is the identity element of $S^{G P}$; thus $S^{N_{n} G}$ is a nilpotent group of class $n$. To see that $\left(\epsilon, S^{N_{n} G}\right)$ is universal with respect to this property, it is enough to show that, for any other such compactification $(\psi, X)$ of $S, \psi^{*}(C(X)) \subseteq N_{n} G$, where $\psi^{*}$ is the dual mapping of $\psi$. If $g \in C(X)$ then $\psi^{*}(g) \in G P$, and

$$
\left(\mu\left[\mu_{1}, \mu_{2}, \cdots, \mu_{n+1}\right]\right)\left(\psi^{*}(g)\right)=g\left(\pi\left(\mu\left[\mu_{1}, \mu_{2}, \cdots, \mu_{n+1}\right]\right)\right)=g(\pi(\mu))=\mu\left(\psi^{*}(g)\right),
$$

where $\pi:\left(\epsilon, S^{G P}\right) \rightarrow(\psi, X)$ is the canonical homomorphism whose existence is guaranteed by the universal property of $\left(\epsilon, S^{G P}\right)$; hence $\psi^{*}(g) \in N_{n} G$, as claimed.

Remarks. 1. As mentioned for $G P$, by the Ellis's theorem [4], we have

$$
N_{n} G \cap W A P=N_{n} G \cap S A P,
$$


and it follows from the fact that $S^{S A P}$ is a topological group, that each side of $(*)$ (and so $N_{n} G$, when $S$ is compact ) consists precisely of those $f \in S A P$ such that for arbitrary elements $s_{1}, s_{2}, \cdots, s_{n+1}$ of $S,\left[R_{s_{1}}, R_{s_{2}}, \cdots, R_{s_{n+1}}\right](f)=f$; hence for a nilpotent group ( of class $n$ ), each side of (*) is equal to $S A P$, and so for a compact nilpotent group $S, N_{n} G(S)=C(S)$.

2. ( The case $n=1$ ). By the theorem, in this case we obtain the universal abelian group compactification. Trivially $N_{1} G \subseteq S A P$; hence by the above characterization of $(*)$ we have

$$
N_{1} G=\{f \in S A P: f(s t u)=f(s u t) \text { for all } s, t, u \text { in } S\}
$$

so for abelian semigroups, $N_{1} G=S A P$, and this can fail to hold if the abelian hypothesis is dropped, as the following examples demonstrate:

For $m \geq 3$, let $D_{2 m}=\prec a, b \mid a^{m}=b^{2}=(a b)^{2}=1 \succ$ be the discrete dihedral group of degree $m,[8]$. A direct computation, using $(* *)$, shows that

$$
N_{1} G\left(D_{6}\right)=\left\{f \in C\left(D_{6}\right): f(1)=f(a)=f\left(a^{2}\right) \text { and } f(b)=f(a b)=f\left(a^{2} b\right)\right\},
$$

and

$$
\begin{gathered}
N_{1} G\left(D_{8}\right)=\left\{f \in C\left(D_{8}\right): f(1)=f\left(a^{2}\right), f(a)=f\left(a^{3}\right), f(b)=f\left(a^{2} b\right),\right. \text { and } \\
\left.f(a b)=f\left(a^{3} b\right)\right\} ;
\end{gathered}
$$

but $S A P\left(D_{2 m}\right)=C\left(D_{2 m}\right)$.

Some other straightforward facts about $N_{1} G$ are as follows:

(i) Using (**), a function $f \in S A P$ is in $N_{1} G$ if and only if $f$ (each finite product of elements of $S)=f$ (each re-ordering of it);

(ii) $N_{1} G$ has a unique invariant mean;

(iii) $N_{1} G$ is the closed linear span of the continuous characters of $S$ (using the Peter-Weyl theorem [5, 22.17], for $S^{N_{1} G}$ ); and

(iv) The $N_{1} G$-compactification of a semidirect product of $S$ and a semitopological semigroup $T$ (in the sense of [2, sec. 5.2]) is isomorphic to a semidirect product of an $F$-compactification of $S$ and the $N_{1} G$-compactification of $T$, for which $F \subseteq N_{1} G(S)$ (with the equality holding in the direct product case).

3. Trivially $N_{n} G \subseteq N_{n+1} G$, and the inclusions can be proper. For example, $N_{1} G\left(D_{8}\right)$ is properly contained in $N_{2} G\left(D_{8}\right)=C\left(D_{8}\right)$ (recall that $D_{8}$ is nilpotent of class 2).

4. $N_{n} G$ is defined by right translates, analogously we can define a space $L N_{n} G$ in terms of left translates (used for constructing the universal left topological nilpotent group compactification ); a natural question that arises is whether the equality $N_{n} G=L N_{n} G$ holds. Of course, for $n=1$ this equality holds.

Some questions concerning the properties of $N_{n} G$, such as left introversion, right amenability, the equality $N_{n} G=L N_{n} G$ and the inclusion $N_{n} G \subseteq W A P$, for $n \geq 2$ are therefore left undecided in this paper; for example we don't know anything about $N_{2} G \subseteq W A P$ in the familiar case of the discrete group of integers. It would be desirable to answer these, at least in the locally compact group setting.

In the end we would like to gratefully acknowledge a research grant from Ferdowsi University of Mashhad. 


\section{REFERENCES}

[1] L. Auslander and F. Hahn, Real functions coming from flows on compact spaces and concepts of almost periodicity, Trans. Amer. Math. Soc. 106 (1963), 415-426. MR 26:1871

[2] J. F. Berglund, H. D. Junghenn and P. Milnes, Analysis on Semigroups: Function Spaces, Compactifications, Representations, Wiley, (1989). MR 91b:43001

[3] K. de Leeuw and I. Glicksberg, Applications of almost periodic compactifications, Acta. Math. 105 (1961), 63-97. MR 24:A1632

[4] R. Ellis, Locally compact transformation groups, Duke Math. J. 24 (1957), 119-126. MR 19:561b

[5] E. Hewitt and K. A. Ross, Abstract Harmonic Analysis I. Springer-Verlag, Berlin, (1963). MR 28: 158

[6] H. D. Junghenn, Distal compactifications of semigroups, Trans. Amer. Math. Soc. 274 (1982), 379-397. MR 84h:43017

[7] I. Namioka, Right topological groups, distal flows and a fixed point theorem, Math. Systems Theory 6 (1972), 193-209. MR 47:5166

[8] D. J. S. Robinson, A Course in The Theory of Groups, Springer-Verlag, Berlin, (1982). MR 84k:20001

Department of Mathematics, Ferdowsi University of Mashhad, P.O. Box 1159-91775 MASHHAD, IRAN

E-mail address: vishki@toos.um.ac.ir

E-mail address: pourabd@toos.um.ac.ir 